\title{
Development and validation of fast and simple flow injection analysis-tandem mass spectrometry (FIA- MS/MS) for the determination of metformin in dog serum
}

\author{
Deborah Michel $^{1}$, Matthew Gaunt ${ }^{2}$, Terra Arnason ${ }^{3}$ and Anas El-Aneed ${ }^{1 *}$
}

\author{
${ }^{1}$ College of Pharmacy and Nutrition, University of Saskatchewan, Saskatoon, SK, Canada, S7N 5E5 \\ ${ }^{2}$ Western College of Veterinary Medicine, University of Saskatchewan, Saskatoon, SK, Canada, S7N 5B4 \\ ${ }^{3}$ College of Medicine, University of Saskatchewan, Saskatoon, SK, Canada, S7N 5E5 \\ * To whom correspondence should be addressed \\ Anas El-Aneed \\ College of Pharmacy and Nutrition, University of Saskatchewan, Saskatoon, SK, S7N 5E5 \\ Phone: 306-966-2013; Fax: 306-966-6377; e-mail: anas.el-aneed@usask.ca
}

\begin{abstract}
A simple, fast and sensitive quantification method for the drug metformin in dog serum was developed using flow injection analysis (FIA)- tandem mass spectrometry (MS/MS). The method was fully validated according to industry standards. It is the first time that FIA-MS/MS for metformin was developed surpassing all existing methods in terms of time of analysis. The quantification method was dependent on the formation of $[\mathrm{M}+\mathrm{H}]^{+}$using electrospray ionization (ESI) and employing multiple reaction monitoring (MRM) using quadrupole- linear ion trap (4000 QTRAP ${ }^{\circledR}$ ) instrument. A deuterated internal standard (IS) of metformin bearing six deuterium atoms was used to compensate for matrix effects and for variation in ion current within the ESI source. The ion transitions that were monitored were $\mathrm{m} / \mathrm{z} 130.1 \rightarrow \mathrm{m} / \mathrm{z} 71.0$ and $\mathrm{m} / \mathrm{z} 130.1 \rightarrow \mathrm{m} / \mathrm{z} 60.1$ for metformin and $\mathrm{m} / \mathrm{z}$ $136.0 \rightarrow \mathrm{m} / \mathrm{z} 77.0$ for the internal standard. A linear response $(\mathrm{r}=0.9966)$ was established for a range of concentrations of 5- $2340 \mathrm{ng} / \mathrm{ml}$. The inter- and intra-day variations were within the acceptable criteria for all quality control samples. The method was successfully applied for measurement of serum metformin concentration in dogs after intravenous injection.
\end{abstract}

\section{Introduction}

Re-purposing of established pharmaceuticals has the advantage over de novo drug development in great savings of time and millions in cost. Of recent interest is the potential repurposing of metformin, an oral insulinsensitizer used as first-line treatment for Type 2 diabetes (DM2). Specifically, there is now interest in validating the several meta-analyses indicating the usefulness of metformin in cancer therapy and/or prevention [1-3]. Indeed, cytotoxic effects of metformin in many cancer cell lines has been demonstrated $[4,5]$. In addition to in vitro studies, in vivo evaluation was conducted using the mouse xenograft model system, evaluating tumor growth such as volume and metastases [6].

An emerging animal model for cancer research is the companion canine, particularly in the spontaneously arising and relatively common hematological malignancy, lymphoma. Metformin is not used in dogs, even to treat diabetes, and tolerability and toxicity have been based on accidental ingestion case reports[7]. The most common clinical side effect reported in dogs is vomiting, while less frequent signs include lethargy, diarrhea, hypothermia, hypotension, pale mucous membranes and hind limb tremors[7]. While little is known about metformin's action in dogs, use in other species provides a basic knowledge of its metabolism. Metformin is excreted unchanged in the urine of humans and animals[7-9]. As such, normal kidney function is imperative for the excretion of this drug and to prevent accumulation in the blood. Metformin's activity in dogs will be evaluated to allow for optimized administration and monitoring during ongoing research trials evaluating its efficacy in cancer prevention and treatment.

To obtain the plasma concentration of metformin in dogs, quantitative analysis is needed. There are numerous methods that can measure metformin within biological fluids. These include capillary 
electrophoresis[10, 11] and high performance liquid chromatography (HPLC)[12-14]. The latter being the most widely applied technique and is routinely linked to tandem mass spectrometry (MS/MS). LC-MS/MS is considered the "gold standard" for quantitative analysis[15, 16] due to its superior performance in terms of sensitivity and selectivity. By applying multiple reaction monitoring (MRM) during quantitative analysis, MS can contribute to both the isolation and the detection of target analytes.

LC-MS/MS has been successfully applied for the quantification of metformin in plasma[12, 17, 18]. Variation within these methods primarily focuses on the LC conditions such as the type of column being used or the solvents used in the mobile phase. For example, Bai et al.[19] utilized a cyano column (CN) using gradient elution while Liu and Coleman[20] used hydrophilic interaction liquid chromatography (HILIC) using isocratic conditions. The lower limit of quantification was $0.5 \mathrm{ng} / \mathrm{ml}$ in the latter study[20] while it was $10 \mathrm{ng} / \mathrm{ml}$ when using CN column[19]. In addition, additives are usually added to the mobile phase to enhance the LC separation and peak shape so that quantitative data can be obtained. Such additives may in some occasions interfere in MS analysis by competing with the target analytes during ionization resulting in undesired ion suppression[21].

Flow injection analysis (FIA)- MS, also termed loop injection, is an alternative approach to LC-MS/MS in which the analytical column is removed and detection/separation occurs within the MS instrument [22-24]. In such cases, analyte response rather than chromatographic separation is used for quantification. FIA-MS/MS was successfully used in the simultaneous quantification of nine macrolides extracted from beef, poultry and pork muscles [23]. The latter study utilized a guard column rather than an analytical one. However, Nanita et al. quantified six pesticides extracted from agricultural products without the need for any column prior to injecting the sample into the mass spectrometry [25]. While generally speaking, matrix effects are expected to increase once the analytical column is removed, Buse et al.[26] showed that the removal of the analytical column was beneficial as tri-ethanol amine (TEA), used in LC-MS/MS as ion pairing reagent[21], was no longer needed. The use of TEA resulted in undesirable ion suppression[21] which was eliminated when the analytical column was removed [26]. However, to achieve quantitative data, method development was needed including changes within the mobile phase.

Despite existing LC-MS/MS methods for the quantification of metformin[12, 17-20], all methods require relatively long run time and the use of analytical column. The removal of the analytical column can substantially increase speed of analysis and simplify the acquisition of quantitative data. To our knowledge, there is no published work in which FIA-MS/MS was used for the quantification of metformin. In this paper, we demonstrate the development of fast and simple FIA-MS/MS quantification method that was successfully applied for the quantification of metformin within dog serum.

\section{Experimental}

\subsection{Materials and Apparatus}

Metformin (97\% purity) was purchased from Sigma-Aldrich (Oakville, ON, CA). Metformin-D6 (99.8\% D enrichment) was purchased from Medical Isotopes Inc. (Pelham, NH, US) as the internal standard. Formic acid was purchased from EMD (Gibbstown, NJ, US). Water and Acetonitrile LCMS grade solvents were purchased from Fisher (Ottawa, ON, CA).

\subsection{FIA-MS-MS conditions}

FIA-MS/MS was performed using a 1200 Agilent (Mississauga, ON, CA) High Performance Liquid Chromatography (HPLC) system interfaced to an AB Sciex 4000 hybrid triple quadropole linear ion trap mass spectrometer (Concord, ON, CA) equipped with a Turboionspray ${ }^{\mathrm{TM}}$ interface. Applied Biosystems/MDS Sciex Analyst software (Version 1.6.0) was used for system control and quantification.

A sample volume of $2 \mu \mathrm{L}$ was injected using the 1200 Agilent autoinjector set to $4{ }^{\circ} \mathrm{C}$ and was delivered with an isocratic mobile phase consisted of water/acetonitrile (30:70, v/v) with $0.1 \%$ formic acid at a flow rate of $0.2 \mathrm{~mL} / \mathrm{min}$ for a run time of 2 minutes. Multiple reaction monitoring (MRM) was achieved by using electrospray ionization (ESI) source in the positive ion mode. The monitored precursor ion $\rightarrow$ product ion transitions for 
metformin were $\mathrm{m} / \mathrm{z}$ 130.1 $\rightarrow \mathrm{m} / \mathrm{z} 71.0$ and $\mathrm{m} / \mathrm{z} 130.1 \rightarrow \mathrm{m} / \mathrm{z} 60.1$ and for the internal standard metformin-d6 m/z $136.0 \rightarrow \mathrm{m} / \mathrm{z} 77.0$.

The source temperature was set to $400^{\circ} \mathrm{C}$, ion spray voltage (ISV) $5500 \mathrm{~V}$, curtain gas (CUR) 40 , nebulizer gas (GS1) 40, heater gas (GS2) 40, collision gas (CAD) 6 and using an exit potential of 10 for all transitions. The declustering potential (DP), collision energy (CE), collision exit potential (CXP) were optimized to $41 \mathrm{~V}, 33 \mathrm{~V}$ and $12 \mathrm{~V}$ respectively for metformin while the internal standard parameters were $46 \mathrm{~V}, 31 \mathrm{~V}$ and $14 \mathrm{~V}$ respectively. Dwell time for all transitions was $150 \mathrm{msec}$ at unit resolution. Nitrogen was used as the gas for all cases and the interface heater was on.

\subsection{Standard preparation}

Stock solutions of the analyte and the internal standard were prepared at a concentration of $779.8 \mathrm{ug} / \mathrm{mL}$ and $788 \mathrm{ug} / \mathrm{mL}$ respectively in water/acetonitrile $(20: 80, \mathrm{v} / \mathrm{v})$. All stock solutions were stored at $4{ }^{\circ} \mathrm{C}$. Working stocks for the standard was made by diluting the stock metformin in water/acetonitrile $(20: 80, \mathrm{v} / \mathrm{v})$ to seven standard points in a range from $0.1 \mu \mathrm{g} / \mathrm{mL}$ to $51.0 \mu \mathrm{g} / \mathrm{mL}$. Standards were prepared by adding $10 \mu \mathrm{L}$ of each standard to $40 \mu \mathrm{L}$ of pooled blank serum and gently mixed. To each standard, $170 \mu \mathrm{L}$ of precipitation solution (acetonitrile containing $0.1 \%$ formic acid and $20 \mathrm{ng} / \mathrm{mL}$ metformin-d6) was added. Each standard was vortexed for 30 seconds and then centrifuged at $14,000 \mathrm{rpm}$ at $4{ }^{\circ} \mathrm{C}$ for 10 minutes using Beckman Allegra 25R centrifuge. The supernatant was transferred to HPLC vials.

\subsection{Sample preparation}

Dog serum samples were thawed at room temperature and gently mixed by inversion. Samples were prepared by adding $170 \mu \mathrm{L}$ of precipitation solution to $50 \mu \mathrm{L}$ of serum sample. Each sample was vortexed for 30 seconds and then centrifuged at $14,000 \mathrm{rpm}$ at $4{ }^{\circ} \mathrm{C}$ for 10 minutes. The supernatant was transferred to HPLC vials.

\subsection{Method validation}

The method validation followed the FDA Guidance for Bioanalytical Method Validation [27], including matrix effects, selectivity, linearity, precision, accuracy, recovery, reproducibility, and stability. Matrix effects was evaluated by comparing the instrument response of the IS added to the extracted dog serum sample in comparison to the IS directly injected in the mobile phase. The selectivity ensured no interference of other components from the sample matrix at the level of lowest limit of quantification (LLOQ) as demonstrated by analyzing six different dog serums.

A standard curve of seven points, ranging from $5 \mathrm{ng} / \mathrm{mL}$ to $2340 \mathrm{ng} / \mathrm{mL}$, was constructed by determining the best fit of peak-area ratios (peak area ratio of the analyte to internal standard) versus the analyte concentration. A linear regression analysis weighing the standard curve with $1 / \mathrm{X}^{2}$ was applied. The performance of the curve was assessed by evaluating deviation of standards from the nominal concentration and evaluating the slope, intercept and coefficient of determination ( $\mathrm{r}$ ) of the curve. Precision and accuracy of the standard curve were determined by replicate sets of quality control sample (QC). Accuracy of LLOQ was accepted if $\leq 20 \%$ of the nominal value and $\leq 15 \%$ of nominal value for the QC samples. While precision of the LLOQ was accepted if $\leq$ $20 \%$ coefficient of variation (CV) and QCs $\leq 15 \%$ CV was met. The interday and intraday accuracy and precision were determined by the performance of the $\mathrm{LLOQ}=5 \mathrm{ng} / \mathrm{mL}$, low QC $(\mathrm{LQC})=14 \mathrm{ng} / \mathrm{mL}$, middle QC $(\mathrm{MQC})=$ $78 \mathrm{ng} / \mathrm{mL}$ and high QC (HQC) = $1950 \mathrm{ng} / \mathrm{mL}$.

Dilution integrity was preformed to prove diluted samples have the same accuracy and precision as undiluted samples. A spiked serum sample at a concentration of $3899 \mathrm{ng} / \mathrm{mL}$ was extracted and then diluted with blank serum to $389.9 \mathrm{ng} / \mathrm{mL}(10 \mathrm{x})$ and $38.9 \mathrm{ng} / \mathrm{mL}(100 \mathrm{x})$.

To examine the recovery of metformin, one set of serum samples were spiked before extraction and another set was spiked after extraction. The recovery was determined by comparing the peak areas for each concentration obtained from both sets. Two different concentrations were evaluated, $12 \mathrm{ng} / \mathrm{mL}$ and $117 \mathrm{ng} / \mathrm{mL}$.

The stability of metformin was tested from 1) bench top, 2) stored in the autosampler, 3) freeze/thaw, and 4) long term stability (55 days) using the QC samples. A set of six replicates of each QC were prepared for each 
stability evaluation. For the bench top stability, QC samples were stored on the bench top at room temperature for 7 hours then the samples were extracted and analyzed. Another set of QC samples were extracted and analysed then stored in the autosampler for 48 hours and reinjected. The freeze/thaw QC samples were stored in the $-80^{\circ} \mathrm{C}$ freezer and were exposed to three freeze/thaw cycles. The samples were allowed to thaw completely and remained at room temperature for at least one hour. Samples were returned to the $-80{ }^{\circ} \mathrm{C}$ freezer for another 24 hour period prior to removal for the next freeze-thaw cycle. The 55 day stability samples were frozen at $-80{ }^{\circ} \mathrm{C}$ for 55 days before thawing samples at room temperature. All samples were extracted as per the sample preparation section.

\subsection{Application}

To investigate the potential of the validated method to pharmacokinetic studies; it was applied to dogs to determine metformin concentrations in serum samples over time. A $28.9 \mathrm{~kg}$ castrated male mixed breed dog was fasted overnight and allowed free access to water. Metformin hydrochloride (Sigma-Aldrich, St. Louis, USA) was reconstituted to $50 \mathrm{mg} / \mathrm{mL}$ with $0.9 \% \mathrm{NaCl}$ (Hospira, Montreal, Canada), drawn through a 0.22 micrometer sterile filter (Millex-GS, Merck, Germany) and administered intravenously $23.4 \mathrm{mg} / \mathrm{kg}$ via a jugular venous catheter. Samples for analysis were collected after removing $2 \mathrm{~mL}$ of blood. Samples were collected at $0,0.33,0.57,1$, 1.33, 1.67, 2, 2.5, 3, 4, 5, 6, 7, 8, 10, 12, 15, 18, 24, 30, 36, 48, and 72 hours after metformin administration. Blood was placed into polypropylene serum tubes, allowed to clot for 45 minutes and then centrifuged at $3500 \mathrm{rpm}$ for 10 minutes. Serum was then separated and stored in clean polypropylene tubes at $-80^{\circ} \mathrm{C}$ until analysis.

\section{Results and discussion}

\subsection{Method Development}

Metformin is a hydrophilic drug that has been successfully separated and quantified using LC-MS/MS [12, 18-20]. Various analytical columns were applied including C18[12, 18], CN[19] and HILIC[20] linked to ESIMS/MS. However, all these methods require relatively long time for the separation step as well as for optimization at the LC end of the analysis. In this work, we opted for the removal of the column applying flow injection analysis (FIA), relying on the mass spectrometer "separation" capabilities. FIA-MS/MS has been successfully applied for the analysis of small organic compounds including pharmaceuticals and pesticides[25, 28]. The MRM mode of the QTRAP system allows for the needed specificity ensuring that the analyte of interest is being monitored and quantified.

The main goal in this work is to develop a simple method that can surpass previous methods in terms of sample analysis and analysis time since pharmacokinetic and bioequivalence studies require the analysis of sometimes thousands of samples. To ensure simplicity, we adapted the simple sample preparation method described by Liu A et al.[20] and Bai et al.[19] that is dependent on protein precipitation. The protein precipitation solution was prepared with a lower concentration of formic acid $(0.1 \%)$ to match the mobile phase conditions. The internal standard was spiked into the precipitation solution to give a concentration of $20 \mathrm{ng} / \mathrm{mL}$ prior to sample addition.

The main method development efforts focused on the optimization of the MS conditions since the LC step has been eliminated and FIA was applied. Regarding instrument parameters, DP and CE were optimized so that suitable fragmentation is obtained while the precursor ion remained abundant allowing for MRM analysis. One inherent challenge in the ESI-MS/MS is matrix effect and the inconsistency in ion current response when using ESI source [29]. To account for such variations, the use of internal standard that has similar physicochemical properties to the analyte and elutes at the same time can ensure the validity of the quantitative data [21]. Therefore, we used deuterated metformin that bears 6 deuterium atoms, which will ensure that no cross talk will occur between the analyte transitions and the internal standard.

Figure 1 shows the MS/MS for both the metformin (Fig 1A) and the internal standard (Fig 1B) as well as the proposed structures for the observed ions. As it can be seen, the precursor ion fragmented producing ions observed at $\mathrm{m} / \mathrm{z} 71$ and 60 as the most abundant ions which were chosen for quantification. Similarly, the MS/MS 
of the internal standard resulted in the formation of the ion observed at $\mathrm{m} / \mathrm{z} 77$ as the base peak which was used for the internal standard.

\subsection{Selectivity and Matrix effects}

A typical chromatogram for the double blank control of dog serum (free of analyte and internal standard) is shown in Figure 2A. No interfering peaks from endogenous compounds were observed at the retention times of the analyte or the internal standard from 6 different dogs (data not shown). The analyte and internal standard appeared at 0.17 minutes with a total data acquisition time (i.e. run time) of 2 minutes (Figure 2B).

Matrix effect was evaluated as per Matuszewski et al.[30] equation

$$
\% \text { Matrix Effect }=\frac{\text { Response post-extracted spiked sample }}{\text { Response non-extracted neat sample }} \times 100
$$

where the post-extracted spiked sample contains the analyte added to extracted blank dog serum. The nonextracted neat sample contains the analyte added to the mobile phase $(70 \%$ acidified acetonitrile with $0.1 \%$ formic acid and 30\% acidified water with $0.1 \%$ formic acid). This was performed in replicates of 6 and the matrix effect was $29.9 \pm 3.81 \%$. It is expected that a column or additional pre-MS sample preparation may help reduce the matrix effect. However, the observed matrix effect was not sufficient to undermine the quantification of metformin for the desired clinical application.

\subsection{Linearity of Calibration curve and sensitivity}

A standard curve of seven points was constructed by determining the best fit of peak-area ratios (peak area ratio of the analyte to internal standard) versus the analyte concentration and running a linear regression analysis weighing the standard curve with $1 / \mathrm{X}^{2}$. The linear regression equation is $\mathrm{y}=0.025 \mathrm{x}-0.00335$. The average correlation coefficient $(\mathrm{n}=3)$ was found to be 0.9966 . The lowest limit of quantitation was $5 \mathrm{ng} / \mathrm{mL}$ and the upper limit of quantitation was $2340 \mathrm{ng} / \mathrm{mL}$.

\subsection{Recovery}

The recovery was calculated as the ratio of the analyte peak area of the pre-spiked serum sample before extraction with the analyte peak area of the post extracted serum sample at equivalent concentrations represented as a percent. Two concentrations were tested, the recovery for $12 \mathrm{ng} / \mathrm{mL}$ was $97.3 \%$ while it was $94.5 \%$ for 117 $\mathrm{ng} / \mathrm{mL}$ samples. Such high recovery values ensure that the majority of the drug is extracted. Efficient extraction facilitates the quantitative analysis, particularly the LLOQ.

\subsection{Accuracy and Precision}

Accuracy and precision were evaluated using QC samples as per the FDA guidelines as described in the methods section. The low QC concentration was within 3 fold of the LLOQ concentration and the high QC was within $80 \%$ of the upper limit of quantitation (ULOQ).

Intra-day precision and accuracy were determined by running the LLOQ, low QC, medium QC and high QC in three separate runs, measuring each run in replicates of six. The precision was reported as CV\% which did not exceed 9.65\% for the four QC levels while accuracy was expressed as percent of the theoretical value ranging between 96.19 to $107.03 \%$ as reported in table 1 . The inter-day validation, using 18 replicates results for each of the four QC samples, yielded a precision between 4.53 to 6.54\% and accuracy ranging from 98.10 to $102.59 \%$ as reported in table 2.

\subsection{Stability}

Bench top stability, auto sampler stability, freeze-thaw stability and long term stability were tested using low QC, medium QC and high QC samples, presented in table 3. For the bench top stability of 7 hours, the precision and accuracy of the QC varied from 3.3 to $11 \%$ and 89.5 to $98.1 \%$ respectively. The stability of the QC samples in the auto sampler resulted in a precision range of 2.3 to $6.5 \%$ and accuracy of 96.4 to $101.9 \%$. The long 
term stability, QC samples frozen at -80 degrees $\mathrm{C}$ for 55 days before extraction, showed the low, mid and high QC samples to both be precise and accurate with the values of precision ranging from 3.0 to $6.6 \%$ and accuracy ranging from $87.7 \%$ to $97.6 \%$. It should be noted, however, that one sample in the low QC samples was lower than specification (80.7\%) and was eliminated from calculations as allowed by the FDA guidelines [27]. The freeze/thaw stability, where the QC samples under went three freeze/thaw cycles, were precise ranging from 3.2 to $8.4 \%$ and accurate ranging from 94.4 to $101.6 \%$.

\subsection{Dilution integrity}

Accuracy and precision was evaluated for dilution integrity. The concentrated sample diluted 10 fold had an accuracy of $94.3 \%$ with a precision of $8.5 \%$ while the concentrated sample diluted 100 fold was $84.8 \%$ and $8.9 \%$ respectively.

\subsection{Application of the method on dogs}

The validated method was successfully applied to a pharmacokinetic study where metformin was interavenously applied to animal subjects. One example is shown in Figure 3 illustrating mean serum concentration vs time profile. The sensitivity and specificity of the assay was adequate to characterize metformin serum concentrations for all evaluated subjects (data not shown). The full pharmacokinetic study including both intravenous and oral application of metformin in dogs will be reported in the close future upon completion of data analysis.

\section{Conclusion}

A new validated high throughput FIA-MS/MS quantitative method of metformin in serum was successfully developed. The method was very simple and fast in comparison to published methods. To our knowledge, this is the fastest quantitative method for metformin. The method was sensitive, robust and specific; the LLOQ in the new method was similar to recently reported values despite using more complicated LC-MS/MS methods in these studies $[17,20]$. The use of analytical column can be tedious and significantly increases run times. The total data acquisition for the current method was merely 2 minutes with metformin appearing at 0.10.3 min (Figure 2). The method was successfully applied to quantify metformin in dog serum (Figure 3). Full pharmacokinetic study will be reported in the close future.

\section{Acknowledgement}

Funding to purchase the QTRAP 4000 instrument were obtained through a Canada Foundation for Innovation (CFI)- Leaders Opportunity Fund. Funding to hire Ms. Michel for three-year term was granted from CFIInfrastructure Operating Fund with matching funds from the College of Pharmacy and Nutrition and the College of Medicine, University of Saskatchewan. The University of Saskatchewan Companion Animal Health Fund provided funding to perform the pharmacokinetic evaluation of metformin in dogs. 
Table 1: Intra-day accuracy and precision

\begin{tabular}{|c|c|c|c|c|c|}
\hline Quality control & Replicates & $\begin{array}{c}\text { Analysis day } \\
\text { (\#) }\end{array}$ & $\begin{array}{l}\text { Observed concentration } \\
(\text { mean } \pm \text { SD; ng/mL) }\end{array}$ & $\begin{array}{c}\text { Precision } \\
(\% \mathrm{CV})\end{array}$ & $\begin{array}{c}\text { Accuracy } \\
(\%)\end{array}$ \\
\hline \multirow[t]{3}{*}{$\overline{\text { LLOQ (5 ng/mL) }}$} & 6 & 1 & $4.96 \pm 0.24$ & 4.8 & 99.1 \\
\hline & 6 & 2 & $5.01 \pm 0.37$ & 7.4 & 100.2 \\
\hline & 6 & 3 & $5.01 \pm 0.32$ & 6.4 & 100.3 \\
\hline \multirow[t]{3}{*}{ LQC (14 ng/mL) } & 6 & 1 & $13.69 \pm 0.59$ & 4.3 & 97.8 \\
\hline & 6 & 2 & $14.11 \pm 1.36$ & 9.7 & 100.8 \\
\hline & 6 & 3 & $13.47 \pm 0.29$ & 2.2 & 96.2 \\
\hline \multirow[t]{3}{*}{ MQC (78 ng/mL) } & 6 & 1 & $77.71 \pm 1.38$ & 1.8 & 99.6 \\
\hline & 6 & 2 & $83.49 \pm 2.15$ & 2.6 & 107.0 \\
\hline & 6 & 3 & $82.88 \pm 3.41$ & 4.1 & 106.3 \\
\hline \multirow[t]{3}{*}{$\begin{array}{l}\text { HQC (1950 } \\
\mathrm{ng} / \mathrm{mL})\end{array}$} & 6 & 1 & $2053.75 \pm 136.01$ & 6.6 & 105.3 \\
\hline & 6 & 2 & $2064.75 \pm 38.79$ & 1.9 & 105.9 \\
\hline & 6 & 3 & $1912.65 \pm 59.74$ & 3.1 & 98.1 \\
\hline
\end{tabular}


Table 2: Inter-day accuracy and precision

\begin{tabular}{cccccc}
\hline Quality control & $\begin{array}{c}\text { Concentration } \\
(\mathbf{n g} / \mathbf{m L})\end{array}$ & Replicates & $\begin{array}{c}\text { Observed concentration } \\
(\mathbf{m e a n} \pm \mathbf{S D} \text {; ng/mL) }\end{array}$ & $\begin{array}{c}\text { Precision } \\
\mathbf{( \% \mathbf { C V } )}\end{array}$ & $\begin{array}{c}\text { Accuracy } \\
\mathbf{( \% )}\end{array}$ \\
\hline \hline LLOQ & 5 & 18 & $4.9 \pm 0.3$ & 6.5 & 98.1 \\
LQC & 14 & 18 & $13.8 \pm 0.9$ & 6.4 & 98.8 \\
MQC & 78 & 18 & $79.2 \pm 3.6$ & 4.5 & 101.5 \\
HQC & 1950 & 18 & $2000.6 \pm 117.7$ & 5.9 & 102.6
\end{tabular}

Table 3: Stability data - quality controls of metformin in dog plasma

\begin{tabular}{|c|c|c|c|c|c|}
\hline Quality control & $\begin{array}{c}\text { Concentration } \\
(\mathrm{ng} / \mathrm{mL})\end{array}$ & Stability & $\begin{array}{l}\text { Observed concentration } \\
(\text { mean } \pm \text { SD; } \mathrm{ng} / \mathrm{mL})\end{array}$ & $\begin{array}{c}\text { Precision } \\
(\% \mathrm{CV})\end{array}$ & $\begin{array}{c}\text { Accuracy } \\
(\%)\end{array}$ \\
\hline \multirow[t]{5}{*}{ LQC } & 14 & $0 \mathrm{~h}$ (for all) & $14.57 \pm 0.56$ & 3.8 & 104.0 \\
\hline & & $3^{\text {rd }}$ freeze-thaw & $13.53 \pm 1.14$ & 8.4 & 96.7 \\
\hline & & 7 h (bench-top) & $13.73 \pm 1.50$ & 11 & 98.1 \\
\hline & & $\begin{array}{l}48 \mathrm{~h} \text { (in- } \\
\text { injector) }\end{array}$ & $14.27 \pm 0.93$ & 6.5 & 101.9 \\
\hline & & 55 days at $-80^{\circ} \mathrm{C}$ & $12.3 \pm 0.4$ & 3.0 & 87.7 \\
\hline \multirow[t]{5}{*}{ MQC } & 78 & $0 \mathrm{~h}$ (for all) & $80.13 \pm 3.11$ & 3.9 & 102.7 \\
\hline & & $3^{\text {rd }}$ freeze-thaw & $79.27 \pm 2.53$ & 3.2 & 101.6 \\
\hline & & 7 h (bench-top) & $79.93 \pm 6.97$ & 9.6 & 93.5 \\
\hline & & $\begin{array}{l}48 \mathrm{~h} \text { (in- } \\
\text { injector) }\end{array}$ & $77.83 \pm 3.75$ & 4.8 & 99.8 \\
\hline & & 55 days at $-80^{\circ} \mathrm{C}$ & $74.3 \pm 4.9$ & 6.6 & 95.2 \\
\hline \multirow[t]{3}{*}{ HQC } & 1950 & $0 \mathrm{~h}$ (for all) & $1910.00 \pm 60.99$ & 3.2 & 97.9 \\
\hline & & $3^{\text {rd }}$ freeze-thaw & $1840.00 \pm 95.08$ & 5.2 & 94.4 \\
\hline & & 7 h (bench-top) & $1745.00 \pm 57.53$ & 3.3 & 89.5 \\
\hline
\end{tabular}


Cite this article as: Michel D, Gaunt MC, Arnason T, El-Aneed A. J Pharm Biomed Anal. 2015 Mar 25 (107): 22935. doi: 10.1016/j.jpba.2014.12.012. (c) 2015. This manuscript version is made available under the CC-BY-NC-ND 4.0 license.

48 h (in-

injector)

$1880 \pm 42.90$

2.3

96.4

55 days at $-80^{\circ} \mathrm{C}$

$1903.3 \pm 68.31$

3.6

97.6

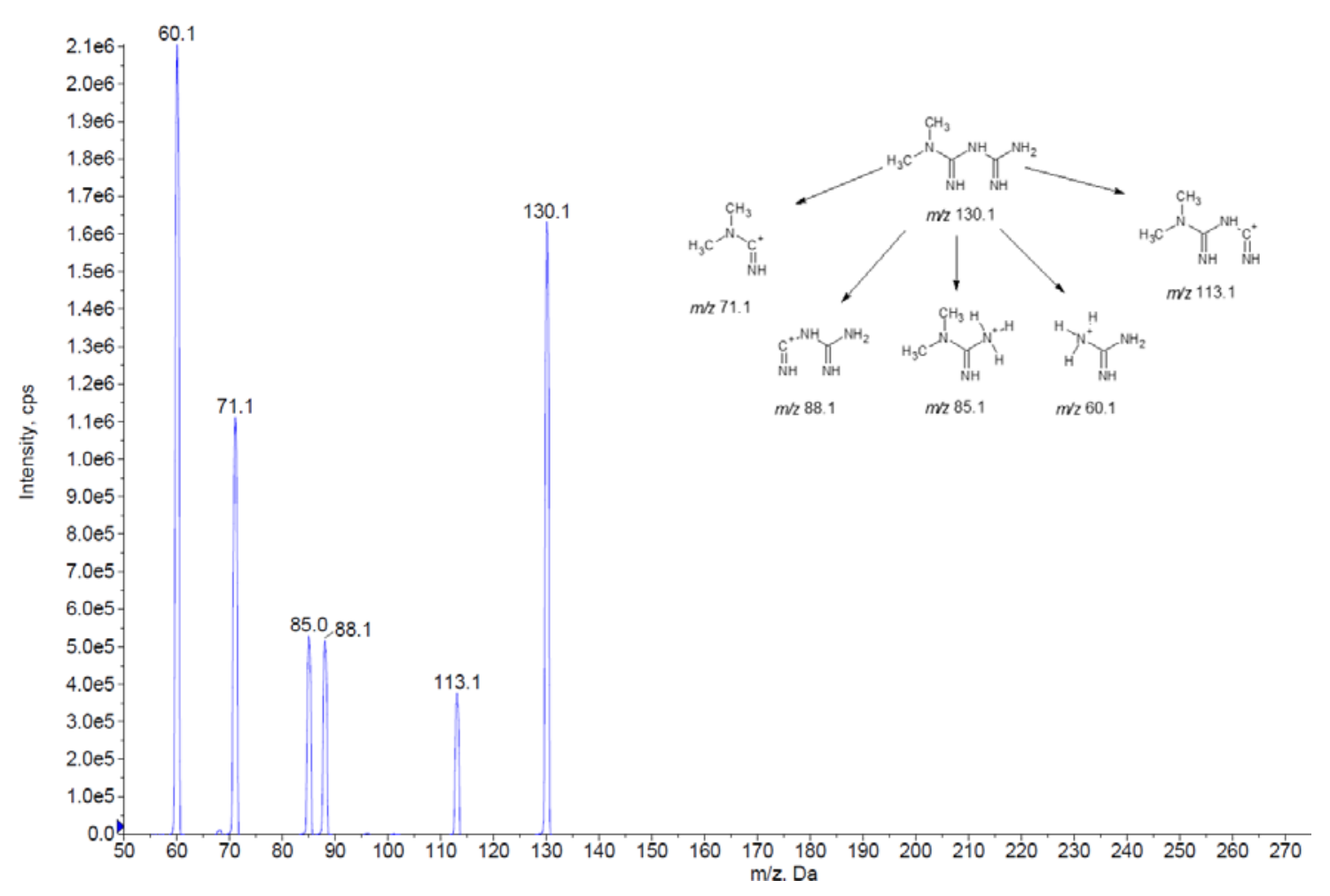


Cite this article as: Michel D, Gaunt MC, Arnason T, El-Aneed A. J Pharm Biomed Anal. 2015 Mar 25 (107): 22935. doi: 10.1016/j.jpba.2014.12.012. (c) 2015. This manuscript version is made available under the CC-BY-NC-ND 4.0 license.

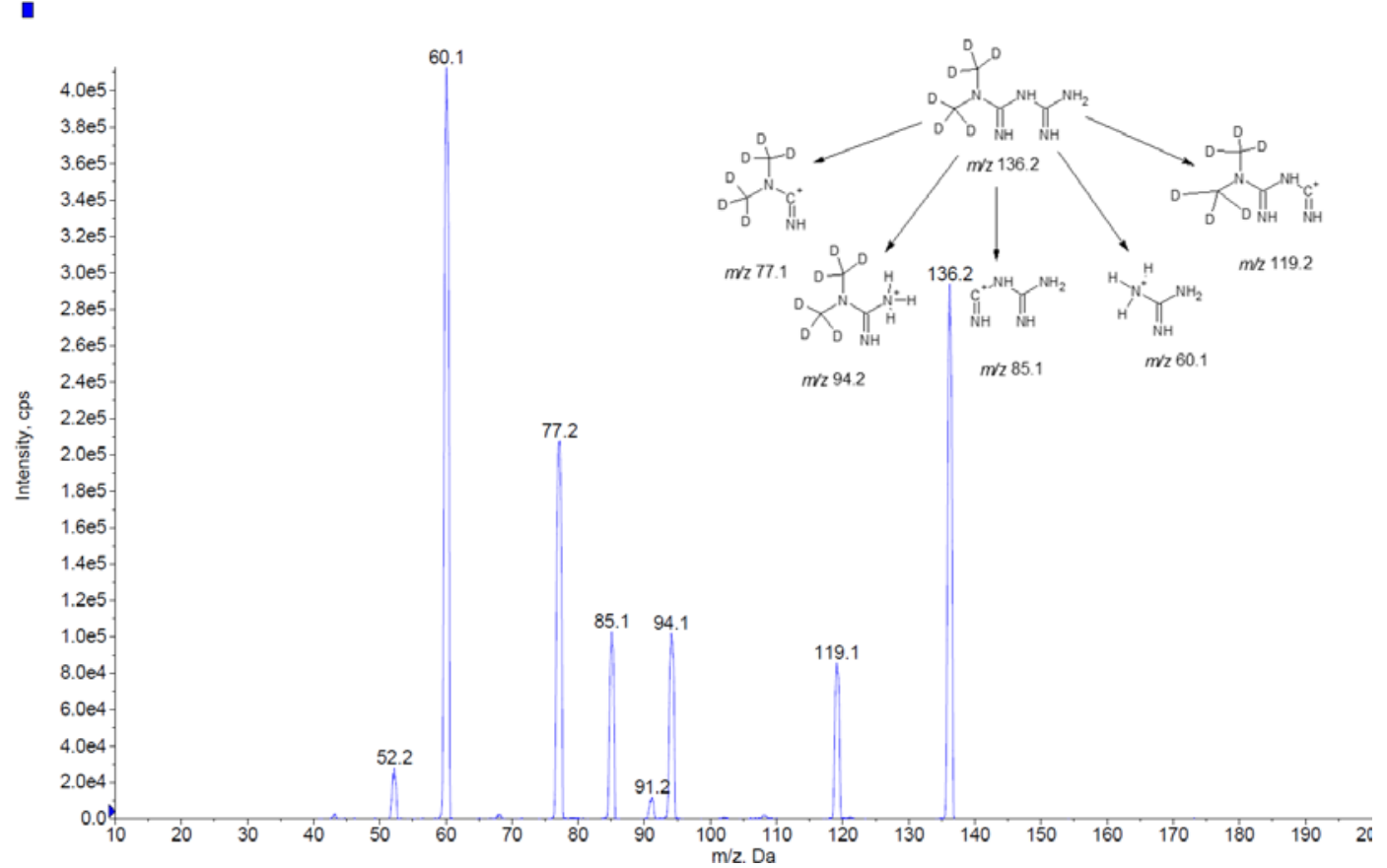

Fig 1: MS/MS of $[\mathrm{M}+\mathrm{H}]^{+}$of metformin (A) and metformin-d6 (B). The fragmentation of each compound are shown as an insert

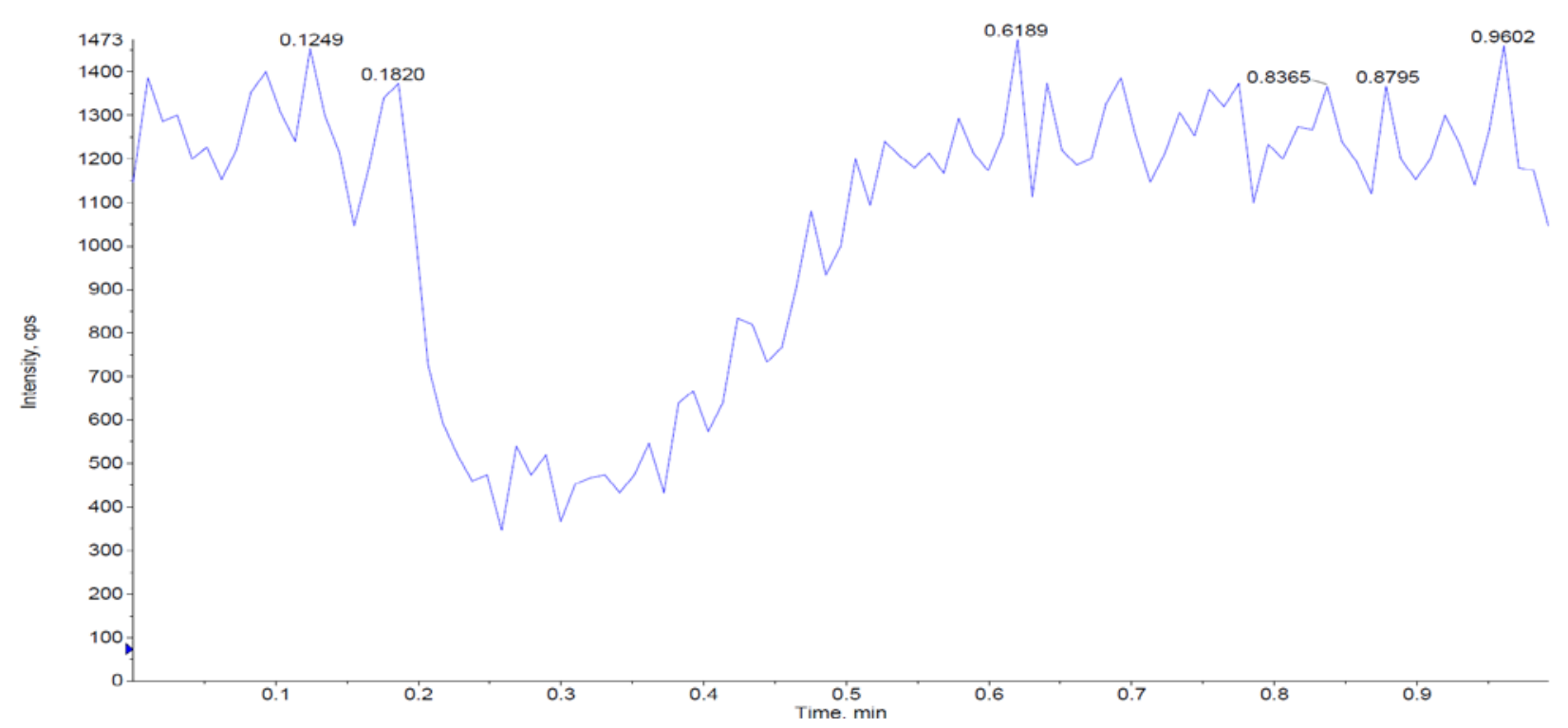




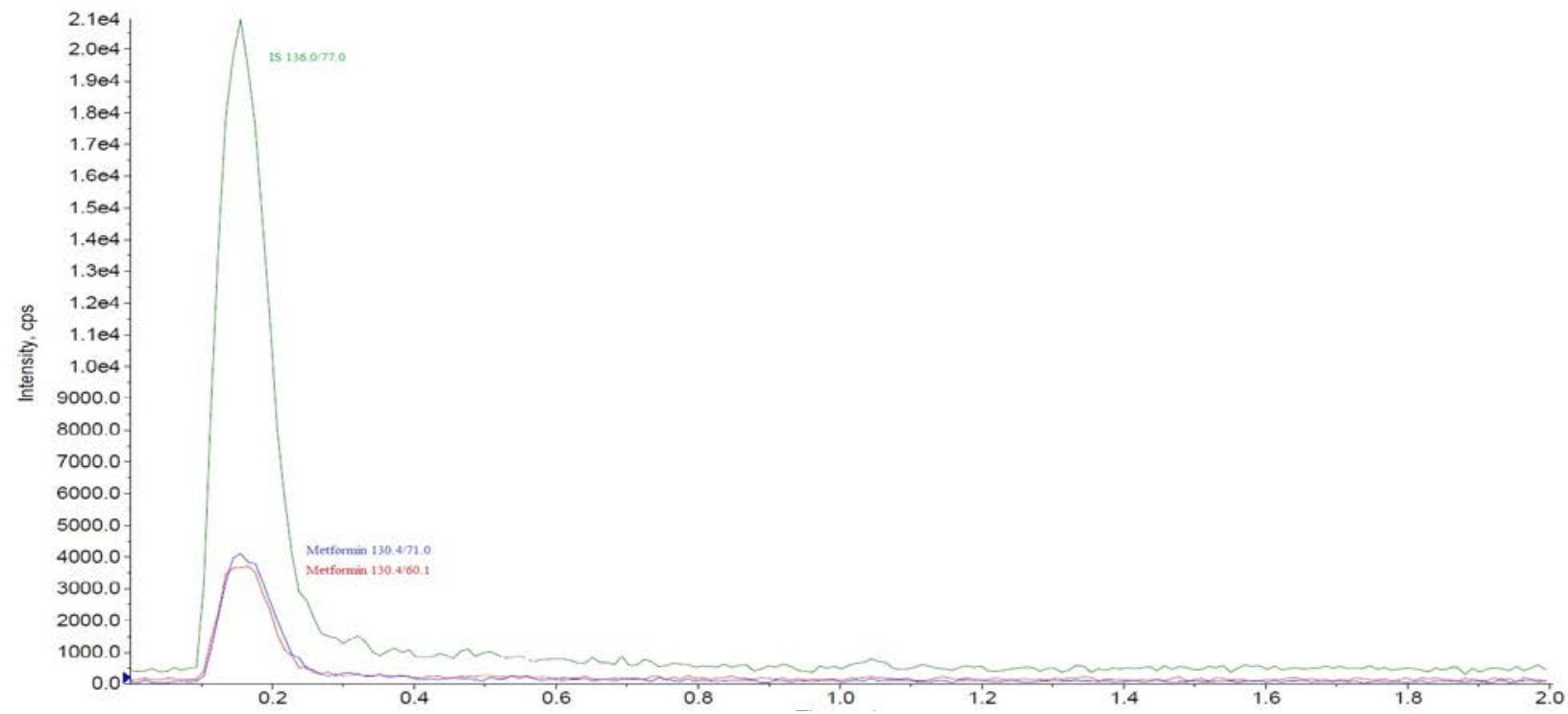

Fig 2: Representative LC-MS chromatograms (A) double blank dog serum and (B) blank dog serum spiked with metformin and internal standard

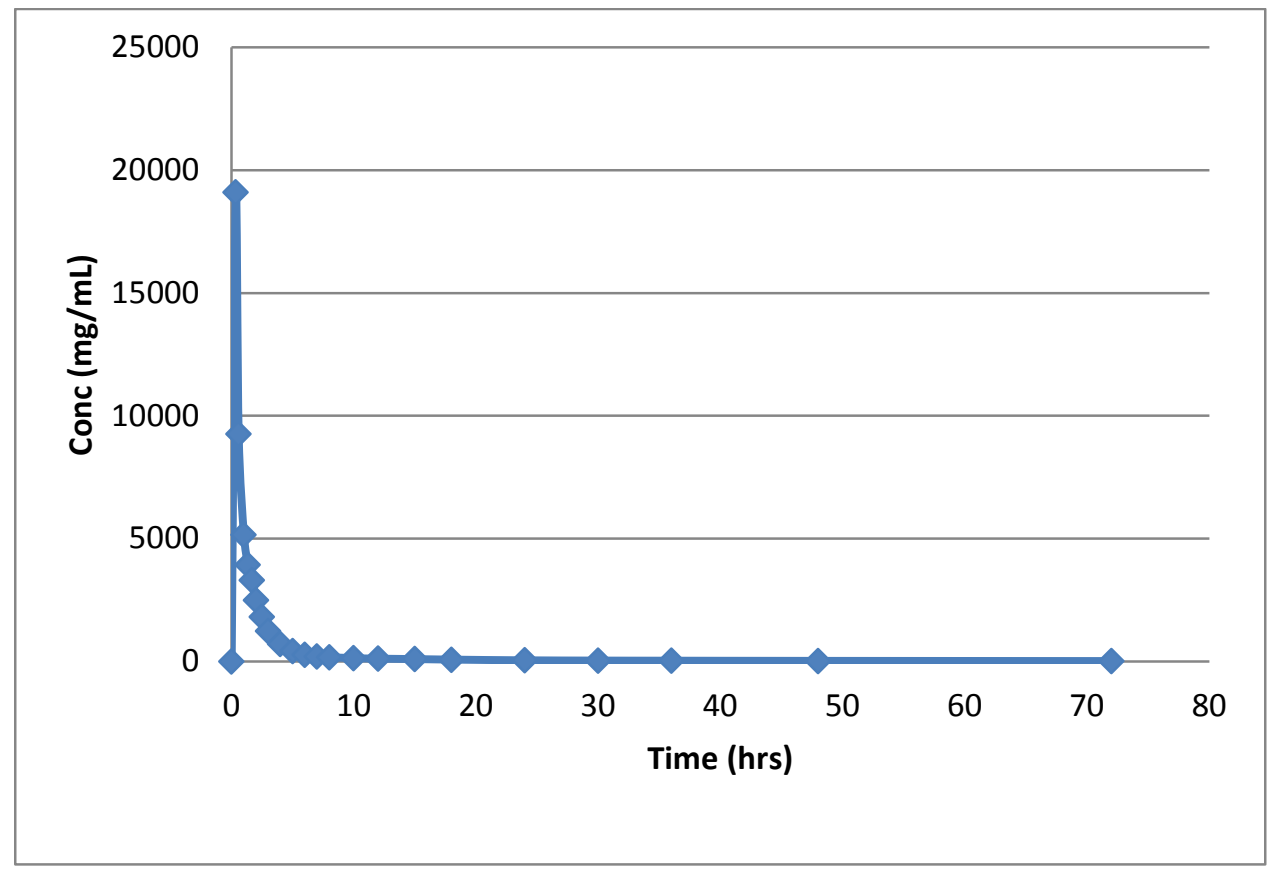

Fig 3: The pharmacokinetic profile for the application of metformin in dog using IV bolus over 72 hour duration. 


\section{References}

[1] G. Libby, L.A. Donnelly, P.T. Donnan, D.R. Alessi, A.D. Morris, J.M. Evans, Diabetes care, 32 (2009) 1620-1625.

[2] M. Bodmer, C. Meier, S. Krahenbuhl, S.S. Jick, C.R. Meier, Diabetes care, 33 (2010) 1304-1308.

[3] H. Noto, A. Goto, T. Tsujimoto, M. Noda, PloS one, 7 (2012) e33411.

[4] D. Iliopoulos, H.A. Hirsch, K. Struhl, Cancer research, 71 (2011) 3196-3201.

[5] H. Liu, C. Scholz, C. Zang, J.H. Schefe, P. Habbel, A.C. Regierer, C.O. Schulz, K. Possinger, J. Eucker, Anticancer research, 32 (2012) 1627-1637.

[6] P. Nangia-Makker, Y. Yu, A. Vasudevan, L. Farhana, S.G. Rajendra, E. Levi, A.P. Majumdar, PloS one, 9 (2014) e84369.

[7] J.B. Heller, Veterinary Medicine, 102 (2007) 231-234.

[8] G.G. Graham, J. Punt, M. Arora, R.O. Day, M.P. Doogue, J.K. Duong, T.J. Furlong, J.R. Greenfield, L.C. Greenup, C.M. Kirkpatrick, J.E. Ray, P. Timmins, K.M. Williams, Clinical pharmacokinetics, 50 (2011) 81-98.

[9] J.L. Hustace, A.M. Firshman, J.E. Mata, American journal of veterinary research, 70 (2009) 665-668.

[10] E.P. Lai, S.Y. Feng, Journal of chromatography. B, Analytical technologies in the biomedical and life sciences, 843 (2006) 94-99.

[11] J.Z. Song, H.F. Chen, S.J. Tian, Z.P. Sun, Journal of chromatography. B, Biomedical sciences and applications, 708 (1998) 277-283.

[12] H.N. Mistri, A.G. Jangid, P.S. Shrivastav, Journal of pharmaceutical and biomedical analysis, 45 (2007) 97106.

[13] N. Koseki, H. Kawashita, M. Niina, Y. Nagae, N. Masuda, Journal of pharmaceutical and biomedical analysis, 36 (2005) 1063-1072.

[14] K. Heinig, F. Bucheli, Journal of pharmaceutical and biomedical analysis, 34 (2004) 1005-1011.

[15] H. Zhang, K. Heinig, J. Henion, Journal of mass spectrometry : JMS, 35 (2000) 423-431.

[16] R. Plumb, J. Castro-Perez, J. Granger, I. Beattie, K. Joncour, A. Wright, Rapid communications in mass spectrometry : RCM, 18 (2004) 2331-2337.

[17] J.G. Swales, R. Gallagher, R.M. Peter, Journal of pharmaceutical and biomedical analysis, 53 (2010) 740-744.

[18] B. Jagadeesh, D.V. Bharathi, C. Pankaj, V.S. Narayana, V. Venkateswarulu, Journal of chromatography. B, Analytical technologies in the biomedical and life sciences, 930 (2013) 136-145.

[19] J. Bai, X. Shi, Y. Du, B. Xiang, S. Wang, D. Cao, Chem. Res. Chinese Universities, 28 (2012) 399-405.

[20] A. Liu, S.P. Coleman, Journal of chromatography. B, Analytical technologies in the biomedical and life sciences, 877 (2009) 3695-3700.

[21] J. Buse, I. Badea, R.E. Verrall, A. El-Aneed, Journal of chromatography. A, 1294 (2013) 98-105.

[22] J. Dankers, J. van den Elshout, G. Ahr, E. Brendel, C. van der Heiden, Journal of chromatography. B, Biomedical sciences and applications, 710 (1998) 115-120.

[23] P.A. Martos, S.J. Lehotay, B. Shurmer, Journal of agricultural and food chemistry, 56 (2008) 8844-8850.

[24] F. Boscaro, G. Pieraccini, G. la Marca, G. Bartolucci, C. Luceri, F. Luceri, G. Moneti, Rapid communications in mass spectrometry : RCM, 16 (2002) 1507-1514.

[25] S.C. Nanita, A.M. Pentz, F.Q. Bramble, Analytical chemistry, 81 (2009) 3134-3142.

[26] J. Buse, R.W. Purves, R. Verrall, I. Badea, H. Zhang, C.C. Mulligan, K.M. Peru, J. Bailey, J.V. Headley, A. El Aneed, Journal of mass spectrometry : JMS, 49 (2014) 1139-1147.

[27] USFDA, Guidance for Industry: Bioanalytical Method Validation. US Department of Health and human Services. Food and Drug Administration, Center for Drug Evaluation and Research (CDER), Center or Veterinary Medicine (CV). 2001.

[28] K. Micova, D. Friedecky, E. Faber, A. Polynkova, T. Adam, Clinica chimica acta; international journal of clinical chemistry, 411 (2010) 1957-1962.

[29] F. Gosetti, E. Mazzucco, D. Zampieri, M.C. Gennaro, Journal of chromatography. A, 1217 (2010) 3929-3937.

[30] B.K. Matuszewski, Journal of chromatography. B, 830 (2006) 293-300. 